H. Fischer, A. Endmann \& M. Krökel (Hrsg.): Mensch und Computer 2015 - Usability Professionals, Berlin: De Gruyter, 2015, S. 408- 414.

\title{
User Journey Mapping
}

\section{Eine Methode im User Experience Design}

\author{
Anja Endmann \\ itCampus Software- und Systemhaus $\mathrm{GmbH}$ - \\ A Software AG Company \\ Nonnenstraße 37 \\ 04229 Leipzig \\ a.endmann@itcampus.de
}

\author{
Daniela Kessner \\ itCampus Software- und Systemhaus $\mathrm{GmbH}$ - \\ A Software AG Company \\ Nonnenstraße 37 \\ 04229 Leipzig \\ d.kessner@itcampus.de
}

\begin{abstract}
Unternehmen interessieren sich zunehmend für eine positive User Experience: Der Nutzer soll die Arbeit mit der Applikation reibungslos und angenehm erleben. Am Anfang von User Experience (UX) Projekten stellen sich uns als UX Consultants häufig folgende grundlegenden Fragen: Wie erhalten wir Einblick in die verschiedenen Nutzerprozesse ${ }^{39}$ ? Wie erheben wir ihre wesentlichen Schritte und Etappen, sowie das jeweilige Nutzererlebnis? Wie erkennen wir, an welchen Stellen im Prozess Research durchgeführt werden sollte? Das User Journey Mapping wurde in einem Tutorial als Methode zum Einstieg in ein Projekt vorgestellt und an einem praktischen Beispiel durchgeführt.
\end{abstract}

\section{Keywords}

Usability, User Experience, User Research, Design, User Journey Map, Methode

\section{Einleitung}

Als UX Consultants arbeiten wir in UX Projekten, in denen Anwendungen entweder vollständig neu entwickelt, optimiert oder erweitert werden sollen. Unsere Projekte sind dabei von unterschiedlicher Dauer und Reichweite, sowie in verschiedenen Branchen angesiedelt, wie beispielweise dem Gesundheitswesen, Automotive und Transportation. Unsere Projektrealität besteht dementsprechend aus häufig wechselnden Themen und Auftraggebern.

39 Ein Nutzerprozess beschreibt alle von einem bzw. mehreren Nutzer auszuführenden Tätigkeiten auf einem User Interface, um ein angestrebtes Ergebnis zu erreichen. 
Unsere größten Herausforderungen am Anfang eines jeden neuen Projekts sind, sich schnell in das Themengebiet unseres Auftraggebers einzuarbeiten, einen umfassenden Überblick über die Projektausgangslage zu gewinnen und relevante erste Schritte im nutzerzentrieten Gestaltungsprozess (ISO 9241-210) identifizieren und angehen zu können. Diesen Überblick benötigen wir schon, bevor gezielte User Research Aktivitäten durchgeführt werden können.

Wir haben deshalb eine Methode gesucht, die einen effektiven und effizienten Einstieg in das Thema ermöglicht, und dabei Stakeholder und Wissensträger von Beginn an aktiv einbezieht.

\section{Definition des User Journey Mappings}

Unsere Methode „User Journey Mapping“ hat ihren Ursprung im „Story Mapping“ (Röpstroff \& Wiechmann 2012; Patton 2014), das im agilen Entwicklungskontext bekannt und bewährt ist. Wir haben das Story Mapping in sieben Kundenprojekten unterschiedlicher Branchen eingesetzt und dabei kontinuierlich zur Methode des User Journey Mappings weiterentwickelt.

Eine User Journey Map bildet einen Nutzerprozess ab mit:

- Dessen Trigger und Ergebnis

- Allen auszuführenden Tätigkeiten

- Beteiligten Personas ${ }^{40}$

- Zuordnung bereits bekannter Anforderungen, Use Cases, Supporttickets, Probleme, etc.

Das User Journey Mapping ist eine kreative Methode, die im Rahmen eines durch UX Professionals moderierten Workshops eingesetzt wird. An diesem Workshop sollten möglichst alle für das Projekt relevanten Stakeholder und Wissensträger, wie der Product Owner, der Produktmanager, Marketing-Mitarbeiter, Softwarearchitekten, Entwickler und Nutzer-Vertreter, teilnehmen.

Wir setzen das User Journey Mapping in unserer Arbeit im Rahmen eines KickoffWorkshops neuer Kundenprojekte ein. Die Methode ist für eine Gruppengröße von vier bis 12 Teilnehmern geeignet. Üblicherweise lässt sich ein durchschnittlich komplexer Nutzerprozess in ca. zwei bis vier Stunden erarbeiten.

40 Eine Persona ist ein prototypischer Nutzer mit Eigenschaften und Nutzungsverhalten einer realen Nutzergruppe. 


\section{Vorteile und Herausforderungen beim Einsatz}

\section{Vorteile}

In unserer Arbeit konnten wir bisher folgende Vorteile der Methode identifizieren:

- Sie bietet UX Professionals einen guten und schnellen Einstieg in ein Projektthema.

- Ein Prozess wird im Überblick visualisiert.

- Der Prozessablauf wird gemeinsam erarbeitet.

- Einzelne Tätigkeiten des Prozesses können im Detail analysiert werden, wodurch Aufgaben identifizierbar werden.

- Es wird identifizierbar, welche Aufgaben im Research untersucht werden sollten.

- Alle Stakeholder werden involviert, fühlen sich gehört und können ihre Meinungen/Ideen in das Projekt einbringen.

- Für UX Professionals im Consulting Bereich bieten Ergebnisse dieser Methode eine gute Möglichkeit, relevante nächste Schritte im nutzerzentrieten Gestaltungsprozess (ISO 9241-210) identifizieren und angehen zu können.

\section{Herausforderungen}

Folgende Herausforderungen der Methode konnten wir identifizieren:

- Der Moderator sollte einige Erfahrung im Moderieren kreativer Prozesse in Gruppen haben, da es nötig werden kann, die Gruppe „,bei Laune zu halten“.

- Der Moderator sollte darauf achten, dass die Gruppe auf die Fragestellung fokussiert bleibt und zum Beispiel kein Ideenworkshop gestartet wird.

- Für jeden im Projekt zu betrachtenden Nutzerprozess entsteht eine eigene User Journey Map.

- Werden mehr als drei Nutzerprozesse betrachtet, sollten die Berührungspunkte der Prozesse in einer eigenen Visualisierung erarbeitet werden (siehe nachfolgendes Kapitel).

\section{Vorgehen zur Erstellung einer User Journey Map}

\section{Setting}

Eine gute Vorbereitung ist unerlässlich, besonders dann, wenn der Workshop in der Kundenorganisation stattfindet. Es muss vorab sichergestellt werden, dass ein ausreichend 
großer Raum zur Verfügung steht, der Bewegungsfreiheit gewährt und mit einer großen Pinnwand ausgestattet ist. Auf einem breiten Stück Packpapier an der Pinnwand entsteht die User Journey Map. Weitere kreative Materialien wie Flipchart, Klebeband, Filzstifte und Klebezettel in verschiedenen Farben dürfen nicht fehlen.

\section{Durchführung}

Damit ein Workshop zur Erstellung einer User Journey Map durchgeführt werden kann, müssen die für den Nutzerprozess relevanten Personas, sowie aktuelle Nutzungsprobleme dieser Nutzergruppe bekannt sein. Hilfreich ist es auch, wenn bereits bekannte Anforderungen, etwa themenrelevante Supporttickets, in den Workshop mitgebracht werden.

Das Vorgehen beim Erstellen einer Journey Map kann in neun Schritten beschrieben werden:

\section{Schritt 1: Detaillierung der Personas}

Hierfür werden die für das Projekt relevanten Personas benannt und für jede Persona ein Flippchart-Papier vorbereitet. Jeder Teilnehmer sammelt dann auf Klebezetteln einer Farbe Pains und auf Zetteln einer anderen Farbe Gains für jede Persona. Dabei steht auf jedem Klebezettel genau ein Fakt. In der Gruppe werden die Inhalte reihum vorgestellt und an das Flipchartpapier geheftet.

\section{Schritt 2: Vorbereitung der Journey Map}

In der Gruppe werden der Prozessname, Prozesstrigger und Prozessergebnis, sowie die Namen der Personas auf Klebezettel einer neuen Farbe notiert. Die Prozessangaben werden als Überschrift an die vorbereitete Pinnwand geklebt, die Personas werden vertikal am linken Rand angepinnt.

\section{Schritt 3: Notieren der zum Prozess gehörigen Tätigkeiten}

Jeder Teilnehmer notiert nun für sich auf Klebezettel einer neuen Farbe alle aus seiner Perspektive relevanten Tätigkeiten, die beim Erreichen des Prozessziels zu erledigen sind. Pro Klebezettel wird genau eine Tätigkeit erfasst.

\section{Schritt 4: Organisieren der Tätigkeiten}

Jeder Teilnehmer stellt seine Inhalte der Gruppe vor und platziert dabei seine Klebezettel an der Pinnwand, entlang einer gedachten, von links nach rechts verlaufenden Zeitachse. In der Vertikalen werden die Tätigkeiten der ausführenden Persona zugeordnet. Während alle Teilnehmer ihre Inhalte nacheinander vorstellen, können Duplikate aufeinander geklebt und ähnliche Inhalte in einer Spalte zusammengeführt werden.

\section{Schritt 5: Aufgaben identifizieren und benennen}

Die Gruppe schaut nun gemeinsam auf die User Journey Map. Jetzt können Inhalte weiter verdichtet und die Abfolge diskutiert und angepasst werden. Tätigkeiten, die ein ähnliches 
Teilziel haben, werden zu Aufgaben gruppiert. Die Gruppe vergibt einen Aufgabennamen, der auf einem Klebezettel einer weiteren Farbe benannt wird.

\section{Schritt 6: Tätigkeiten und Aufgaben ergänzen}

Nachdem die Tätigkeiten zu Aufgaben zusammengefasst wurden, überprüft die Gruppe die entstandene User Journey Map mit folgenden Fragestellungen: Fehlen Tätigkeiten/Aufgaben? Welche Situationen gibt es, die einen anderen Ablauf erfordern? Wie sieht dieser alternative Ablauf aus? Fehlende Tätigkeiten /Aufgaben werden an der entsprechenden Stelle der User Journey Map ergänzt.

\section{Schritt 7: Zuordnung der Pains und Gains}

In der Gruppe werden zunächst die Gains einer jeden Persona der betreffenden Aufgabe im Prozess zugeordnet. Gegebenenfalls können neue Gains ergänzt werden.

Anschließend ordnet die Gruppe die Pains der einzelnen Personas den betreffenden Aufgaben zu. Wieder können neue Pains ergänzt werden. Zur Identifikation weiterer Pains kann folgende Fragestellung unterstützen: Sind weitere Herausforderungen/Probleme bekannt, die im Nutzungskontext auftreten?

Häufig kommen an dieser Stelle in der Gruppe spontan Design- oder Lösungsideen auf. Diese können auf Klebezettel einer weiteren Farbe notiert und in die User Journey Map integriert werden.

\section{Schritt 8: Hinzufügen vorhandener Dokumente}

Liegen im Projekt bereits Anforderungen, Supporttickets, Use Case Dokumente, dokumentierte Nutzungsprobleme etc. vor, können diese ebenfalls in der User Journey Map an entsprechender Stelle ergänzt werden.

\section{Schritt 9: Identifikation der nächsten Schritte}

Abschließend überprüft die Gruppe im Plenum die entstandene User Journey Map: Wo im Prozess gibt es viele Hinweise auf Nutzungsprobleme? Wo im Nutzungsprozess fehlen Informationen? Auf diese Weise identifiziert die Gruppe, an welchen Stellen der User Journey gezielte User Research Aktivitäten durchgeführt werden müssen, beziehungsweise, für welche Aufgaben bereits mit der Entwicklung von Designkonzepten begonnen werden kann. 


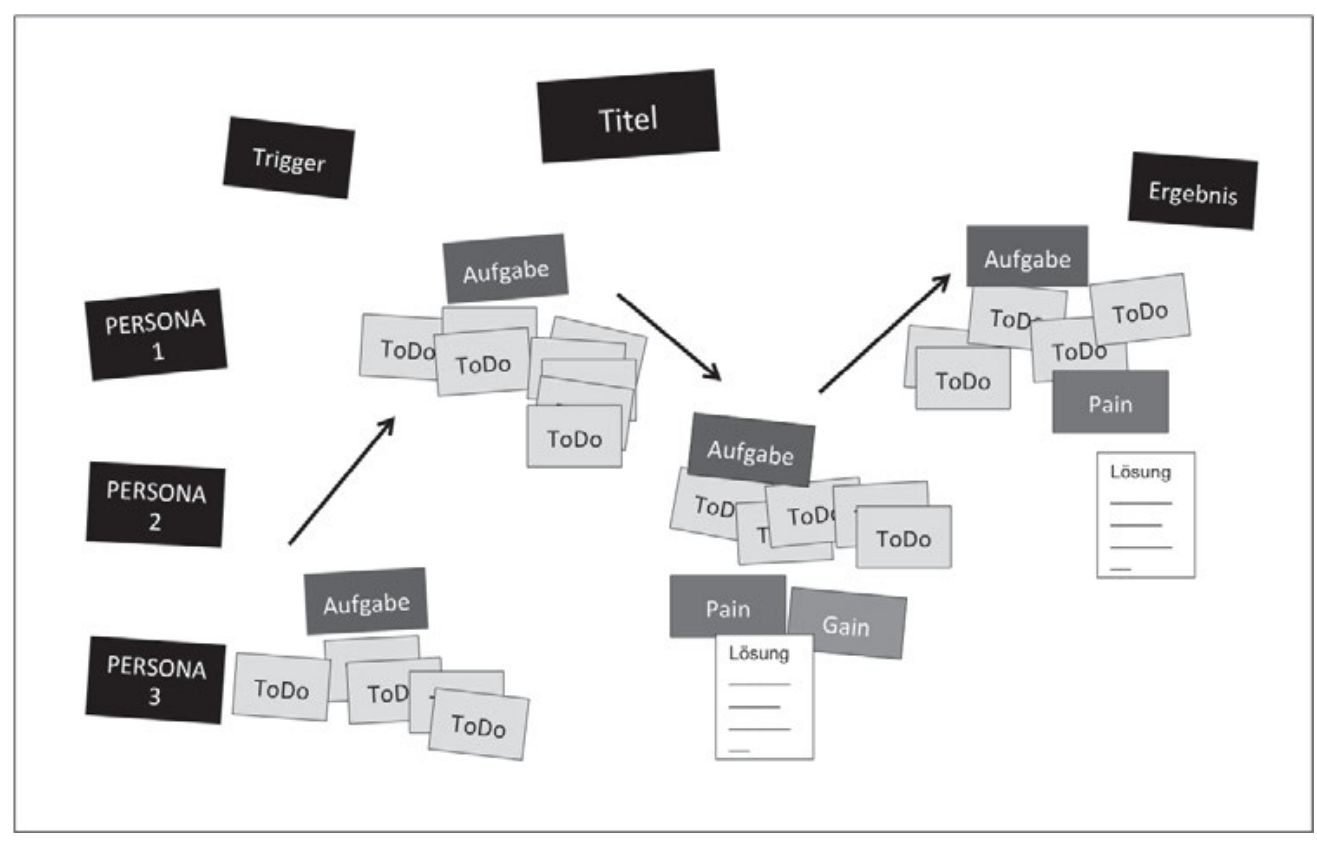

Abbildung 1: Schematische Darstellung einer User Journey Map

\section{Wenn mehrere Nutzerprozesse betrachtet werden sollen}

In den meisten unserer Projekte gilt es, mehr als einen Nutzerprozess abzubilden. Hat man es mit mehr als drei Prozessen $\mathrm{zu}$ tun, besteht eine neue Herausforderung darin, etwaige Zusammenhänge der verschiedenen Prozesse miteinander zu identifizieren und abzubilden.

In diesen Fall wird eine separate Pinnwand für die Prozesslandkarte vorbereitet, auf der die projektrelevanten Nutzerprozesse in einer Übersicht dargestellt werden. Die Prozesslandkarte wird vor den einzelnen User Journey Maps erstellt.

An der Pinnwand wird der Name der Anwendung als Überschrift notiert. Die Gruppe sammelt die relevanten Nutzerprozesse und notiert für jeden den Namen, Trigger, und das Ergebnis. Die einzelnen Prozesse werden anschließend chronologisch von oben nach unten horizontal angebracht. In jeder Zeile stehen links der Trigger, mittig der Prozessname und rechts das Ergebnis. Sind alle Prozesse so abgebildet, werden die Schnittstellen/ Berührungspunkte zwischen ihnen visualisiert.

Jetzt priorisiert die Gruppe die Prozesse und beginnt mit der Erstellung der User Journey Map für den wichtigsten Prozess. 


\section{Zusammenfassung}

Das User Journey Mapping ist eine kreative Methode für den schnellen Einstig in komplexe UX Projekte. Sie erlaubt es uns, noch vor der Durchführung von Research-Aktivitäten in kurzer Zeit und unter Beteiligung aller Stakeholder, die für das Projekt relevanten Nutzerprozesse kennenzulernen und die im Projekt notwendigen UX Aktivitäten zu identifizieren und zu planen. Die Durchführung auf einem Kickoff-Workshop nimmt ca. vier Stunden in Anspruch und verlangt eine/n erfahrene/n Moderator/in, um die Teilnehmer in der Erstellung der Journey Map anzuleiten. Aus der fertigen Map wird sowohl konkreter Research-Bedarf, wie auch die Priorisierung für Design-Konzepte abgeleitet.

\section{Literatur}

DIN EN ISO 9241-210 (2010). Ergonomie der Mensch-System-Interaktion- Teil 210: Prozess zur Gestaltung gebrauchstauglicher interaktiver Systeme. Beuth, Berlin.

Patton, Jeff (2012). User Story Mapping: Discover the Whole Story, Build the Right Product. O'Reilly \& Associates, Sebastopol.

Röpstroff, Sven und Wiechmann, Robert (2012). Scrum in der Praxis: Erfahrungen, Problemfelder und Erfolgsfaktoren. dpunkt.verlag, Heidelberg. 